\title{
PROTECTING TRANSMISSIONS WHEN USING POWER CONTROL ON 802.11 AD HOC NETWORKS
}

\author{
Alexandre Andrade Pires and José Ferreira de Rezende \\ GTA - COPPE \\ Universidade Federal do Rio de Janeiro, Brazil \\ Email: (andrade, rezende)@gta.ufrj.br
}

Carlos Cordeiro

Philips Research USA

Briarcliff Manor, NY 10510

Email: Carlos.Cordeiro@philips.com

\begin{abstract}
This paper presents the ALCA (Asymmetric Link Collision Avoidance) protocol. ALCA was designed to deal with a known deficiency of the Basic Scheme Gomez et al., 2001 for power control in 802.11 ad hoc networks, which occurs when links become asymmetrical as a result of power control. The proposed ALCA mechanism conveys transmission duration information to these terminals through a simple modification of the 802.11 MAC protocol. Through extensive simulation, the performance of ALCA is investigated and compared to PCM (a solution that requires major hardware updates). Results indicate that ALCA outperforms PCM while being a considerably less complex solution.
\end{abstract}

Keywords: $\quad$ ad hoc, power control, IEEE 802.11

\section{Introduction}

A number of proposals have been made in order to reduce power consumption in ad hoc networks so as to provide longer autonomy to mobile nodes, and hence increase the network lifetime. Among the areas of vigorous research, power-aware routing has attracted considerable attention, and is based on the idea of appropriately selecting routes in order to minimize power consumption Narayanaswamy et al., 2002; Gomez et al., 2001; Agarwal et al., 2001; Monks et al., 2001. A complementary research area focuses on designing protocols that save energy through the use of transmit power control. The basic idea is to avoid exceeding the transmission power level needed for link establishment. 
Power control techniques are often based on a feedback mechanism, which is responsible for information exchange between receiver and transmitter so as to adjust transmission power to the minimum necessary level required for link maintenance Agarwal et al., 2001; Monks et al., 2001. Besides saving transmission energy, power control has the advantage of contributing to a potential spatial reuse of the channel. In other words, transmissions at a lower power level are kept in a reduced range, which decreases medium contention and results in a higher aggregated throughput by allowing other transmissions to take place simultaneously in the network. Also, power control reduces co-channel interference, which leads to better signal-to-noise ratio at the receivers.

We can identify three broad approaches to establish a power control loop between transmitters and receivers in IEEE 802.11 ad hoc networks: those employing a separate control channel Lin et al., 2003; those making use of busy tones Monks et al., 2001; and those adding extra information fields in all control and data frames Agarwal et al., 2001. The first two schemes usually give good performance as power control feedback is provided while the transmission is ongoing. On the other hand, these schemes require that two transceivers be available, which make them expensive and complicated solutions. In what concerns the third approach, its main drawback is the introduction of asymmetric links among medium contenders. Asymmetric links increase the likelihood of collisions, putting at risk the benefits obtained from power control.

In this paper, we propose a solution to avoid collisions in the presence of asymmetric links. This scheme, named ALCA (Asymmetric Link Collision Avoidance), has the advantage of being simple, efficient and easily implementable. Through extensive simulations, we have compared ALCA with existing solutions that are based on the same approach, and the results are very promising. The remainder of this paper is organized as follows. Section 1 presents the existing work on power control for IEEE 802.11 ad hoc networks. Next, in Section 2 the ALCA mechanism is described in detail. Section 3 presents the simulation environment and results. Finally, this paper is concluded in Section 4 with some future directions.

\section{Power Control on IEEE 802.11 Networks}

This section describes the major existing schemes that add extra information fields in the IEEE 802.11 frames to perform power control in ad hoc networks.

\section{BS: Basic Scheme}

A simple mechanism for power control on IEEE 802.11 networks is based on power information exchange during the RTS, CTS, DATA and ACK frame exchange Gomez et al., 2001; Agarwal et al., 2001, and constitutes the starting point for several possible enhancements. In short, this technique is based on the 
idea of transmitting the RTS and CTS frames at the maximum transmit power level. This frame exchange is then used to determine the minimum power level needed for a successful communication between the two nodes, thus allowing them to transmit the DATA and the ACK frames with the minimum required power. Clearly, this method does not take any advantage of the potential spatial channel reuse, as the reservation of the channel is always done at the maximum power level. On the other hand, it conserves significant energy as DATA and ACK frames are transmitted at lower power levels.

On the downside, the reduction on the transmit power of the DATA and ACK frames may cause collisions in the receiver (with the DATA frame) as well as in the transmitter (wit the ACK frame). This is due to the introduction of asymmetric links between the communicating nodes and other potential transmitters that cannot sense the medium busy during the ongoing low power transmission. Such collisions result in frame retransmission and hence increased energy consumption, eliminating any possible gain obtained from power control. In the next section we elaborate on this problem and present the PCM protocol Jung and Vaidya, 2002 as a possible solution.

\section{PCM: Power Control MAC}

The virtual carrier sensing mechanism of IEEE 802.11 works well for terminals inside the transmitter's transmission range, where they are able to successfully decode the frame received. However, a problem occurs with the terminals located within the carrier-sensing zone (CS-Zone) of the transmitter, which is characterized by the fact that nodes in this zone can only sense the carrier in the channel, but cannot decode the frames received. As a result, these nodes do not have access to the duration information contained in the frame headers. Considering that propagation effects are the same in both directions, the transmitter is also in these nodes' CS-Zone. In this situation, a collision will take place if any of these terminals decide to initiate a transmission during the ongoing low power transmission. In an attempt to minimize the occurrence of this scenario, the standard specifies that if a node receives a signal with sufficient power to trigger a carrier detection, but not high enough to result in a correct frame reception, it shall set its NAV to an inter-frame space called EIFS (Extended Inter Frame Space) once the carrier detection is over. Nodes within the transmission range and CS-Zone adjust their NAVs during RTS-CTS-DATAACK transmission. Nodes in transmission range correctly set their NAVs when receiving RTS or CTS. However, since nodes in the C-Zone cannot decode the packet, they do not know the duration of the packet transmission. To prevent a collision with the ACK reception at the source node, nodes within the $\mathrm{C}$ Zone set their NAVs for the EIFS duration. The main purpose of the EIFS is to provide enough time for a node to receive the ACK. 
However, when power control is employed the above sequence may fail. For example, consider the Basic Scheme. After a CTS transmission, a terminal located in the CS-Zone of the CTS transmitter will set its NAV with the EIFS value. Next, as the DATA transmission will be carried out at a lower power level there will be a consequent reduction of the CS-Zone. Once the EIFS expires, it may so happen that the terminal senses the medium as idle if it is no longer inside the CS-Zone of the original transmission. In this case, the node concludes that the channel is free and, after the backoff process is over, initiates an RTS transmission.

This problem was studied in Jung and Vaidya, 2002 which shows that this failure may result in a significant increase in the number of collisions and retransmissions. As a result, instead of the desired energy conservation effect, the power consumption in the network would actually increase. To overcome this problem, the authors propose a power control MAC (PCM) scheme that avoids these collisions through a periodic in-frame variation of transmit power level for DATA frames. During the low power DATA frame transmission, the transmitter must advertise nodes within the original CS-Zone (i.e., the zone defined by the power level used for transmission of the RTS/CTS) that the transmission is still going on. To this end, PCM mandates that during the transmission of DATA frames the terminal should raise its transmit power to the maximum power level, triggering carrier detection and further NAV setting to EIFS duration for those terminals in the CS-Zone. After a period of time transmitting at the maximum power level, the power is again reduced to the minimum level. This periodic increase/decrease of the transmit power level continues indefinitely until the DATA transmission is completed, triggering carrier detection at the terminals within the original carrier sensing range and preventing them from accessing the medium and causing collisions. PCM achieves a better energy conservation, as it minimizes collision probability and guarantees that the power saving gain obtained from power control is not eliminated by excessive retransmissions. However, a major drawback with this solution is that it requires transmitters to vary their transmission power very quickly and with high precision.

\section{BSM: Basic Scheme with Memory}

In the Basic Scheme, RTS and CTS frames are always sent at the maximum transmit power. In other words, the power control loop is used for the sole purpose of reducing the DATA and ACK frames transmission power. As an enhancement, the Basic Scheme with Memory (BSM) employs a table at each terminal that maintains the previous transmission power level used by the terminal to communicate with each one of its neighbors. For each MAC address of a neighbor terminal, there is an entry in the table that keeps the exact power 
necessary for communication with that node, which is obtained from the last RTS-CTS-DATA-ACK exchange between the nodes. As opposed to the Basic Scheme, a node uses the reduced power level for all its transmissions, i.e., RTS-CTS-DATA-ACK.

Among the advantages of BSM scheme, we can observe a higher power saving and a higher potential to channel spatial reuse. The channel reuse is enhanced as the union of the CS-Zone for the RTS frame and the CS-Zone for the CTS frame represents the reserved area for a transmission. If these frames are sent at reduced power level, such zones will be reduced, allowing for the occurrence of simultaneous transmissions. On the other hand, this mechanism provides a lower protection for DATA transmissions, possibly increasing the collision probability. Also, the BSM scheme should be enhanced with mechanisms for scenarios with mobility since it does not calculate the needed transmit power in a per-packet basis.

\section{ALCA: Asymmetric Link Collision Avoidance}

As discussed in 1, asymmetric links due to power reduction on DATA and ACK frames may result in frame collisions and further retransmissions, leading to poor performance. This problem is inherent to any mechanism of power control based on the Basic Scheme Gomez et al., 2001; Agarwal et al., 2001. As a result, a solution to the asymmetric link problem is critical and essential to any power control protocol employing high power control frames (i.e., RTS and CTS) for medium reservation and collision avoidance, and reduced power DATA and ACK frames.

The solution proposed in this work, the ALCA (Asymmetric Link Collision Avoidance) protocol, overcomes this problem by allowing terminals in the carrier sensing zone of a transmission to have access to transmission duration information despite the fact that terminals within this zone are not able to correctly receive and decode a frame, and so cannot read the duration field present in RTS and CTS frames. As we have showed in 1, the asymmetric link problem is a consequence of this inability.

Although terminals located in the carrier sensing zone cannot decode the correct type of a packet, but they can detect a data carrier on the channel. Moreover, they can sense with some level of accuracy the instants of time when carrier sense turns on and off. The proposed scheme is based on conveying the Current Transmission Duration Information (CTDI) through the physical duration of the carriers that transport RTS and CTS frames. This way, terminals within the CS-Zone will be able to determine the CTDI, preventing them from accessing the channel until the current transmission successfully terminates.

The physical duration of the RTS/CTS frames can be increased by simply adding a few bits to them. However, it is important to keep in mind that these 
additional bits do not carry any information by themselves, but are just a way to enlarge the frames according to the CTDI. Thus, the ALCA protocol provides a discrete set of $N$ different Carrier Durations (CD) for RTS and CTS frames. For terminals inside the reception zone the $\mathrm{CD}$ is simply ignored, as these terminals are able to access the CTDI included in RTS and CTS duration field. However, terminals located in the CS-Zone use the CD information to extract the CTDI and hence avoid collisions.

Broadly, the ALCA protocol operation is comprised of two major parts. In the first one, a node that is going to initiate a transmission calculates the respective CTDI and stores it in an RTS frame. In addition, it determines which $\mathrm{CD}$ it will have to use in order to allow terminals in the carrier sensing zone to recover the intended CTDI. This selection is made by comparing the CTDI to predefined thresholds. Thus, the transmitter chooses a $C D_{i}$ among those $N$ possible CDs. Finally, the transmitter includes the necessary word of bits in the RTS frame, so that the transmission has the intended duration on the channel (those bits are ignored by nodes inside reception zone). Obviously, to ensure protocol consistency, ALCA requires that all terminals in the network know beforehand both the thresholds as well as the set of possible CDs.

In the second part of ALCA operation, a terminal in the CS-Zone of the considered transmitter senses a data carrier on the channel. As the frame cannot be successfully decoded, the information of signal duration (i.e., the CD) is extracted. In this case, instead of setting the NAV to the standard EIFS value as in IEEE 802.11, the node selects an appropriate value for the EIFS based on the extracted signal duration. Clearly, this value has to be larger than the CTDI calculated using the signal duration in order to avoid a collision. The same protocol operation applies in case of CTS frames so as to convey transmission duration to nodes in the carrier sensing zone of the receiver. Last, but not the last, it is important to note that the ALCA protocol may work in combination with any power control scheme based on the BS methodology.

\section{Simulation Results}

We have used ns-2.26 to perform our simulations. For the evaluation of the power control schemes, we have used random scenarios generated by arbitrary location of 40 nodes inside a specified physical area. A total of 20 terminals are transmitters, while the other 20 are receivers. Although all flows are single hop, but they do not always occur with the closest node to the transmitter. Any node inside of transmitter reception zone can be chosen to be a receiver. Reception zone is a circle of $250 \mathrm{~m}$ of radius, while CS-Zone has a radius of $500 \mathrm{~m}$. We run simulations varying the aggregated rate of the CBR sources. Two metrics are considered: aggregated throughput (in Mbps) and the amount of data transmitted by unit of energy (in Megabytes per Joule). Each point is 
an average of 50 runs, each of them with nodes in different locations. The error bars in the curves correspond to $99 \%$ confidence intervals. Packet size is fixed in 1024 Bytes.

The simulations were run varying the physical area where nodes are placed and the noise power. The noise power, or noise floor, corresponds to the power at the receivers when all transmitters are turned off. We use two values for physical area, $1 \mathrm{~km}^{2}$ and $4 \mathrm{~km}^{2}$. For noise power, we also consider two values, namely, $-100 \mathrm{dBm}$ (the typical noise of office environments) and $-83 \mathrm{dBm}$ (which corresponds to a noisy environment). Our goal is to study the behavior of the schemes with respect to network densities and noise variations. Finally, we have focused on three configurations: large area with low noise, large area with high noise, and small area with low noise.

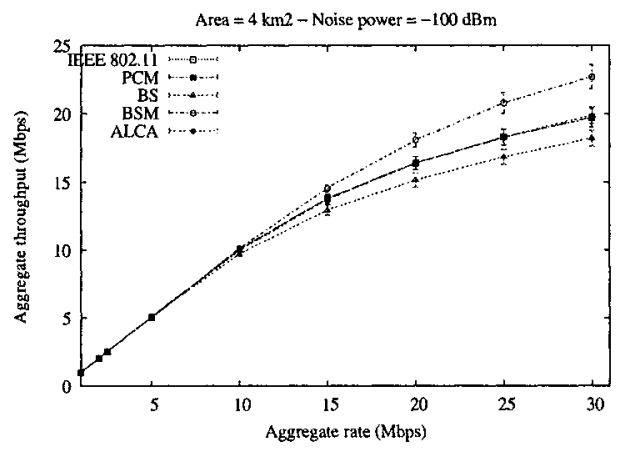

Figure 1. Sparse network with low noise - Aggregate throughput.

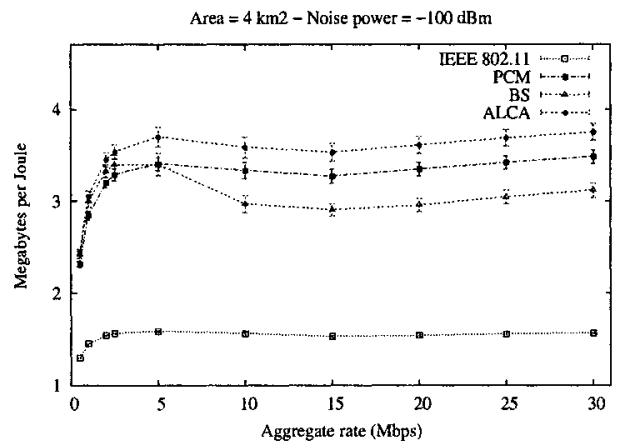

Figure 2. Sparse network with low noise - Energy efficiency. 
Figures 1 and 2 present aggregated throughput and Megabytes per Joule when using an $4 \mathrm{~km}^{2}$ area and noise power of $-100 \mathrm{dBm}$. We can see that there is minimal difference in aggregated throughput achieved by the five schemes and that they are equivalent with respect to data delivery, under these conditions. Aggregated throughput was evaluated for each configuration, always resulting in similar curves: small differences in averages, always smaller than error bars. While the maximum aggregated offered load was $30 \mathrm{Mbps}$ (on a 11 Mbps channel), which corresponds to a $1.5 \mathrm{Mbps}$ CBR source on each transmitter, we conclude that full network capacity is never reached. This explains why aggregated throughput curves for the schemes are similar, even if collisions and retransmissions occur more or less frequently in each one of them. As aggregated throughput curves are always similar, we now discuss only energy related curves for the other two configurations.

From Figure 2, we note important differences amongst the schemes. Pure IEEE 802.11 has the worst performance. PCM and BS, on the other hand, are seen to outperform IEEE 802.11. In low offered loads, BS is more efficient in terms of energy savings. However, as CBR load increases so does the asymmetrical link problem, hence causing collisions and consequent performance degradation. With its periodic variation of transmit power, PCM triggers EIFS in the CS-Zone and is thus able to avoid the asymmetrical link problem and achieve higher energy conservation as compared to BS. Clearly, the ALCA protocol works well in solving asymmetrical link problem without the need to increase the transmit power level of data frames, thus achieving superior energy conservation.

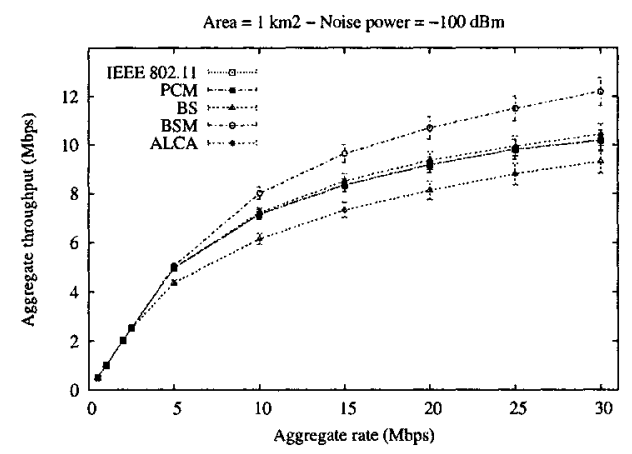

Figure 3. Dense network with low noise - Aggregate throughput.

Figures 3 and 4 present results of reduced physical area, with low noise. As area is reduced and network density increases, channel contention is higher and we can see that the point where BS and PCM schemes have similar performance occurs in reduced rates in contrast to the large area, low noise config- 


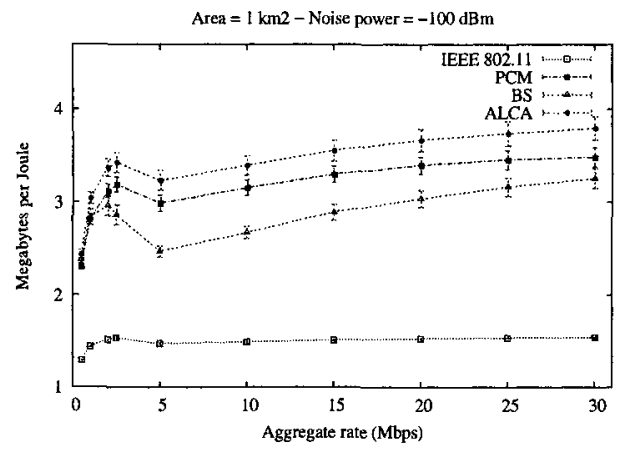

Figure 4. Dense network with low noise - Energy efficiency.

uration. As the total number of collisions increases, relative gain of ALCA is reduced when compared to PCM, but it still achieves superior performance.

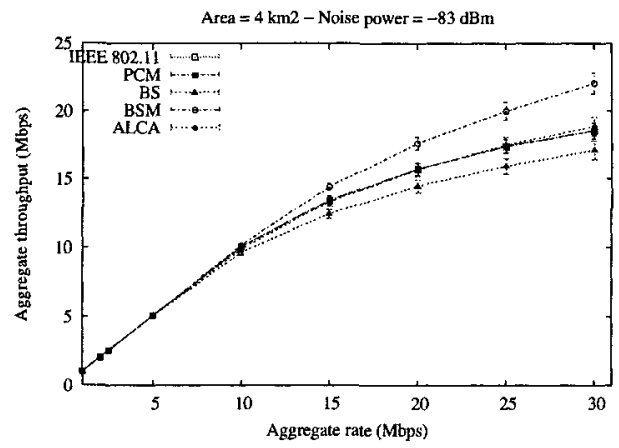

Figure 5. Sparse network in a noisy environment - Aggregate throughput.

Figures 5 and 6 investigates the scenario when the area is large $\left(4 \mathrm{~km}^{2}\right)$ and noise raises to $-83 \mathrm{dBm}$, and reflects numerous situations such as industry plants. Once more, ALCA exhibits best performance while we can see that PCM follows ALCA more closely, as noise increases the occurrence of collisions.

\section{Conclusions}

This work has presented the ALCA (Asymmetrical Link Collision Avoidance) protocol, which is intended to solve asymmetrical link problem, a known deficiency of Basic Scheme (BS) based power control mechanisms. ALCA was evaluated together with BS which supplies power control while ALCA 


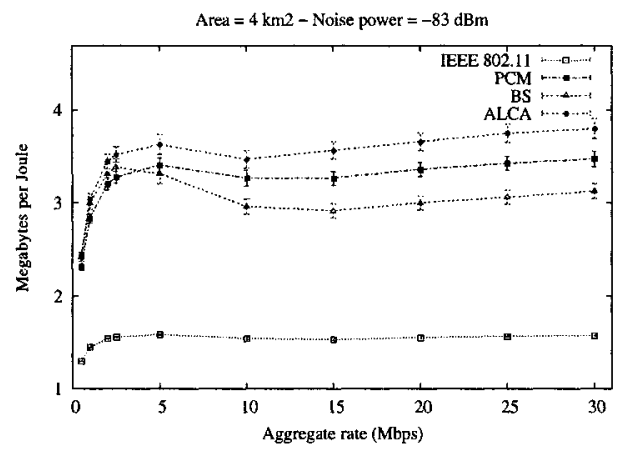

Figure 6. Sparse network in a noisy environment - Energy efficiency.

works on solving asymmetrical link problem. Therefore, ALCA can be used in conjunction to any other power control protocol based on the BS method.

The results confirm that asymmetrical link problem can increase the number of collisions and retransmissions, possibly canceling the energy saving obtained by power control. Additionally, results show that ALCA protocol presents a good performance in dealing with asymmetrical link problem. ALCA has experienced energy savings slightly superior to PCM, and much superior than other schemes. Therefore, we can conclude that the ALCA protocol provides higher energy conservation than other existing schemes without the need for drastic changes in hardware or in the 802.11 MAC protocol. As future work, we plan to evaluate ALCA behavior in a mobile network environment and analyze its performance when used with other power control schemes. Also, we will evaluate the impact of the coexistence of devices supporting ALCA with legacy 802.11 devices without any power control.

\section{References}

Agarwal, S., Krishnamurthy, S., Katz, R. H., and Dao, S. K. (2001). Distributed power control in ad-hoc wireless networks. In IEEE PIMRC'01.

Gomez, J., Campbell, A. T., Naghshineh, M., and Bisdikian, C. (2001). Conserving transmission power in wireless ad hoc networks. In ICNP'01.

Jung, E. and Vaidya, N. H. (2002). A power control MAC protocol for ad hoc networks. In $A C M$ MOBICOM'O2.

Lin, X.-H., Kwok, Y.-K., and Lau, V. K. N. (2003). A new power control approach for ieee 802.11 ad hoc networks. In IEEE PIMRC'03.

Monks, J. P., Bharghavan, V., and mei W. Hwu, W. (2001). A power controlled multiple access protocol for wireless packet networks. In IEEE INFOCOM'O1.

Narayanaswamy, S., Kawadia, V., Sreenivas, R. S., and Kumar, P. R. (2002). Power control in ad-hoc networks: Theory, architecture, algorithm and implementation of the COMPOW protocol. In European Wireless 2002. 